\title{
KNAPPHEIT VON SINN. UBER DEN ENERGIEVERBRAUCH KULTURELLER ZEICHENSYSTEME
}

ALBRECHT KOSCHORKE

1.

In diesern Beitrag soll von wpoetischen Energien einem erwartbaren Sinn. Es wird nicht um die Ausdruckskraft des dichterischen Wortes, um clas Glücksgefühl, das große Dichtungen auslösen können, nicht einmal um das einende, kollektive Ilentität stiftende und deshalb auch ideologisch nutzbare Potential von Dichtung gehen. Mit dem intrikaten Verhältnis zwischen Poesic und Politik habe ich mich in anderen Publikationen befasst ${ }^{t}-$ zuletet als Mitherausgeber des Bandes Despoten dichten. Spracbkunst und Gewalt, der sich der seltsamen Schwäche widmet, die gerade Gewalthertscher gegenüber der Wortkunst zeigen: von der Lyrik (Stalin, Mao, Karadžić) über den Roman (Goebbels, Ghaddafi, Saddam Hussein) und das Drama (Mussolini) bis hin zum Opernlibretto (Kim ll-sung). Gewaltherrschaft ist eine prekäre und instabile Machtform, in der paradoxerweise die Macht des Wortes die größte Rolle spielt, und deshalb kanu man von Gewaltherrschern mit noch größerem Recht sagen, was Gneisenau auf eine Aktennotiz des preußischen Königs erwiderte: dass auf Poesie die Sicherheit der Throne gegründet sei. Ergănzen wir in diesem Fall: auf den Verbund von Poesie und Geheimpolizei.

Statt solche Themen zu vertiefen, seien an dieser Stelle einige heuristische Uberlegungen anderer Art angestellt. Sie bringen das Thema der Energie mit einem Begriff von Poesiec in Verbindung, der in einer weiteren und dem etymo logischen Wortsinn näheren Becleutung verstanden wird: als Poiesis, als das Ma chen, genauer das Sich-Machen einer Ciesellschaft im Spiegel ihrer sprachlichen Möglichkeiten. Es ist also nach einem bestimmten Aspekt von gescllschaftlicher Poiesis zu fragen. Denn auch in den Selbstreptäsentationen, über die eine Gesellschaft sich veranschaulichend und vergegenwärtigend auf sich selber bezieht sind poetische Mechanismen am Werk, insofern sie sich auf den Gebrauch vot

Metaphern und anderen thetorischen Techniken stutzen und sich im Medium 
yon Narrativen oder Dramatisierungen bewegen. Diese poietischen Mechanismen haben maßgeblichen Anteil daran, wie sich der Energiehaushalt des gesell. schatitichen Imaginären teguliert.

Gewönlich denkt man in diesem Zusammenhang an die Mobilisierung von Energien - das Aufstacheln von Leidenschaften, die Bündelung der Atfmerk samkeit auf ein großes Ziel hin, die Hebung des Blicks von den Niederungen des Alttags in eine Welt mythischer oder sakmaler Bezüge Ich will mich dem Thema der poetischen Energie diesmal aber nicht von der Seite der Verausgabung her nähern, sondern aus einer eher defensiven Perspektive: nämlich im Hinblick auf den Energieverbratuch kutureller Zeichensystene und das Problem der Kiappheit, das sich damit unmittelbar assoziiert. Meine Leitfrage heißt also: Was passiert, wenn wir das Problem der Energieknappheit nicht nur auf materielle, sondern auch auf informationelle und symbolische Prozesse bezichen? Wie können wir. der Finsicht einen systematischen Stellenwert zuerkennen, dass Basisprozesse der Informationsbeschaffung, Verständigung, der Erzeugung und Stabilisierung von sozialem Sinn zeitraubend und energieintensiv sind? Wie sind dementsprechend die Vorkehrungen zu analysiereu, die Individuen treffen, um den Snergieaufwand threr symbolischen und kommunikativen Welthewältigung zu begrenzen?

Um die Tragweitc dieser Fragestellung zu ermessen, ist es zweckmäßig, den Blick auf eine Nachbardisziplin zu richten: die Ökonomie. Bekanntlich legt die im 20. Jahrhundert domivante neoklassische Wirtschaftslehre ihren Berechnungen die Fiktion des bomo oeconomicus zugrunde, der als rationaler Entscheider und Nutzenmeximiever verstanden wircl. Wichtiger noch ist für unseren Zusanmenhang, dass dieser bomo oeconomicus grundsätzlich jederzcit tiber vollständige Informationen verfügt und ¿lass die Beschaffung der nötigen Information für ihn keine Mühe und keine Kosten verursacht. Gemäß der Efficient Market Hypothesis (EFM) ist er alleio schon deshalb richt auf das mühsame Zusammentragen von Informationen angewiesen, weil der Markt alle relevanten In formationen beteits in den Preisen realisiert hat. Der vollständig inform ierte rationale Akteur arbeitet also idealiter in einem Umfeld, in dem alle relevanten Marktdaten sich für jeder mann offensichtlich in den Preisen widerspiegela; Abweichungen werden, da gewianträchtig, von Spielenn an Markt umgehend ausgegliches.

Auch wenn dieses Modell durch die Konzepte der bounded rationality, der unvollkommenen Märkte und der durch Informationsasymmetrien gekennzeichneten Beziehung zwischen principal und agent erheblich modifizient wurde, sind seine Standardannahmen doch so mächtig geblieben, dass der Ökonom joseph E. Stiglitz noch 200 bei seiner Nobelpreistede die von ihm verfochtene sinfor- mation economics a als einen nfundamental change in the prevailing paradigm within ecouonics bezeichnet. ${ }^{4}$ Dabei hatte schon vor ihm ein anderer Nobelpreisträger, Douglass C. North, die neoklassischen Wirtschaftstheorien ciner fundamentalen Kritik unterzogen und darnit maßgeblich zur Neubegründung dev Institutionenökonomie beigetragen ${ }^{5}{ }^{5}$ In seincm Buch Institutionen, institutionetler Wandel und Wirtschafisteistung setzt sich North von den herkömmlichen Theorien dadurch ab, dass er historische und kulturelle kaktoren in die Analyse wirtschaftlicher Tauschprozesse einbezicht. Den neoklassischen Modellen wirft er vot, die Transaktionskosten und damit die Rahmenbedingungen des Marktgeschehens als Kostenfaktor zu vernachlässigen. Dazu zählen vorrangig die Kosten vou Informatiousbeschaffung beziehungsweise die Kosten, die durch unvollständige, asymmetrische hnformation und durch die in solchen Fälien notwendigen supplementären Strategien zur Erzeugung von Erwartungssicherheit entstehen. Wie in den zeitgenössischen Medientheorien kommen hier also die Übermittlungsuege ton Information in den Blick, geleitet won der Einsicht, dass Informtion nicht instantan, ubiquitãr und unaufwändig (also kostenlos, ohne Zcit. und Energieverbauch) verfügbar ist und dass sie sich nicht gleichmäRig \%wischen den Akteuren verteilt.

Noth verknupft drei Ansätze miteinander: Erstens akzentuiert er in dem wirtschaftstheoretischen Begriffspanr von Transformation (Herstellung und Um. wandlung von Gütem) und Transaktion die Seite der 'Transaktion mitsamt den dazugehörigen Kosten. Ein wesentlicher Teil dieser Kosten ist durch die Notwendigkeit verursacht, sich mit Information über die Marktverhältnisse zu versorgen. Weil jedoch kein Spieler am Markt vollständige Kenntnis von seinen Mitspielern und den Entwicklungen am Markt haben kann, fehli die Berechnungsgrundlage füt ein streng tationales Kalkül. Trotzdem sind die Wahlmöglichkeiten deshalb nicht beliebig, weil sie durch eine Reihe von intermediären Instanzen - Notmen, Ideologien, kulturelle Vorpuägungen, Praxen, Gewohnheiten - eingeschränkt werden. Dex Gebrauch solcher Voreinstellungen ist also nicht Ursache unvoll. ständiger beziehungsweise verfïlschter Information, sonden ein unentbehrlicbes Mittel, un unter Bedingungen des partiellen Nichtwissens gleichwohl handlungs- und entscheidungsfähig zu bleiben. Insbesondere informelle Regelungen tragen dazu dabei, Koordinationsprobleme auf kostengünstige Weise zul lösen; sie verdanken sich rer Tatsache, sdaß bei gegebenen Tauschlosten [...] beide Partner Interesse daran haben, die Kostspieligkeit der Messung zu mizimieren, und daß Tauschrorgänge sich ,automatisch vollziehen $\alpha$ ?

Dies bewegt North zweitens dazu, den Faktor ,Kulturs in die Analyse von Markverhältuissen einzubeziehen. North versteht Keltur als einen »Informationsfiltere - ein sBezugssystem zur Verschlïsselung und Deutung der Informa tion, welche die Sinne dem Gehirn liefern,${ }^{8}$ das die Entscheidungen dex Akteure 
anleitet. Wieder sind es die sinoffziellen Prozesse, denen er die größte Lenkwirkung und Stabilisierungsleistung zuschreibt, und zwar »in dem Sinne, daß die nicht-formgebundene Lösung von Tauschproblemen aus der Vergangenheit in die Gegenwart fortdauert und jene formlosen Beschränkungen zu wichtigen Faktoren einer Dauer im langfristigen gesellschaftlichen Wandel werden läßtu."

Damit verbindet sich nahtlos ein dritter Aspekt, nämlich die Rolle von Institutionen (im weitesten Sinn dieses Wortes ${ }^{10}$ ). Ähnlich wie die deutschen Sozial. anthropologen des 20. Jahrhunderts sieht North die Leistung von Institutionen darin, menschliches Verhalten von der Notwendigkeit zu entlasten, die Rahmen bedingungen von Handlungen immer wieder ad boc herstellen zu müssen. Auch dieser Gedanke hat bei ihm jedoch eine zusätzliche, informationstheoretische Pointe: Institutionen gleichen das Defizit aus, dass die Akteure am Martt in aller Regel nur unvollkommen informiert sind und deshalb auf ein Regelwerk zurückgreifen müssen, das ihre Risiken vermindert:

In einer Welt vollständiger Information bedarf es keiner Institutionen. Bei unvollständiger Information hingegen werden kooperative Lösungen versagen, wenn nicht Institutionen geschaffen werden, die den beteiligten Personen genügend Informationen liefern, um vertragswidriges Fandeln ahnden zu können."

Märkte bestehen nicht aus atomistischen Individuen, die sich allein an ihrem Nutzenkalkül ausrichten; immer schon sind Entscheidungspräferenzen institutionell vorgebahnt, nicht zuletzt durch die Ideen- und Normensysteme, die im Schutz von Institutionen gedeihen und den Akteuren feste Deutungsmuster mitsamt der zugehörigen Sanktionierungsmacht gegenüber Abweichungen $\cdots$ an die Hand geben. ${ }^{12}$ Wegen der Eigengesetzlichkeit und des Beharrungsvermögens von Institutionen kann dies durchaus negative folgen haben, wenn etwa eine fatale Weichenstellung pfadabhängig kollektive Fehlentwicklungen nach sich zieht Die positive Leistung von Institutionen jedoch besteht darin, dass sie Erwartungssicherheit zu reduzierten Informationsbeschaffungskosten herstellen.

Auch andere Wissenschaften ziehen die Standardan uahme einer rationalen Wab auf der Basis vollständiger Information in Zweifel. Von Seiten der Neuropsychologie ${ }^{13}$ wird geltend gemacht, dass a) kein Akteur jemals über vollständige mformationen zur Entscheidungsfindung verfügt; b) die Bntscheidung selbst Teil der vorauszuberechnenden Katusalkette wäre; c) Menschen entscheidungsvereinfachende Vorkehrungen treffen, etwa das sogenannte "satisficing«, das sich mit einer »balbwegs befriedigenden Lösung« begnügt, ${ }^{14} \mathrm{~d}$ ) Entscheider oft nicht einmal genau wissen, was sie wollen, und dies erst in Lauf des Lntscheidungstindungsprozesses definieren; und e) Emotionen als Vereinfacher dienen, unter anderen dadurch, dass sie die Wahmehmung von Risiken einschränken bezichungsweise erhöhen, in jedem Fall aber eindeutiger scheinen lassen. Experimentelle Untersuchungen zeigen, dass Menschen sich mit »Entscheidungs. Heuristiken « ausstatten, ${ }^{15}$ die die Anzahl der berïcksichtigten Merkmale der Alternativen beschränkt (im Grenzfall auf ein einziges), und dass ein solches heuristisches Verhalten mit vertretbarem Aufwand befriedigende Resultate zeitigt, also im Großen und Ganzen ökonomischer als ein komplexeres Entscheidungsverfahren sein dürfte.

Der Gedanke von Douglass North, dass institutionell, historisch und kulturell bedingte sbared mental models das kognitive und kommunikative Verhalten von Individuen stetem, findet also seine Entsprechung in den Automatismen und Wahmehmungsroutinen, in den verkürzenden Verfahren der pattern recognition oder des template matching der Nenrowissenschaften und Verhaltenspsychologie. ${ }^{16}$ In allen Fällen geht es um Abkürzungsverfahren, man könnte auch sagen: um sparprogramme im Hinblick auf die Verausgabung von Zeit und Energie. Wenn man sich vom Idealbild des rationalen Akteurs, der stets den Übebblick über seine lage bewahrt, verabschieder und stattdessen zu einer Anthropologie der Unvollkommenheits übergeht, dann sind solche heuristischen Umgangsweisen mit unvollständigem Wissen unentbehrlick. Dann erschcinen Men schen -.. auch Ökonomen, und sogar Finanzmarktakteure - als ssituierte, d. h. strukturell begrenzte, standortabhängige und endliche Wesen«, die pausenlos »einfache Lösungen für komplexe Probleme entwickeln und atth unvorherge. sehene schwierige Situationen meistern « müssen. ${ }^{17}$ Wo der Schuz der Instinkte fehlt, müssen Automatismen kulturell generiert werden. Musterkennung, Verhaltensschemata, Faustregeln dienen auf elementaren Operationsstufen demsel. ben Zweck wie die voraussetzungsreichen sozialen Einrichtungen, die geschaffen wurden, um die menschliche Unbehaustheit in der Welt durch Außenstabilisierung auszutgleichen.

Hier zeigt sich nun eine überraschende Funktionsäquivalenz zwischen zwei Weisen dex Formung sozialen Lebens, die in weit auseinanderliegenden Bereichen beheimatet zu sein scheinen und von unterschiedlichen Wissenschaften betreut werden: zwischen Institutionen und Narrativen. Auch von den Erzähl. weisen, in denen eine Kultur sich darstelit, kann ja gesagt werden, dass sie dazu dienen, soziale Erwartungen und Erwartungserwartungen zu stabilisieren; dass sie, in fiktionaler Form, Verhaltensformulare entwickeln und dementsprechend die Wahl- und Handlungsmöglichkeiten einschränken - gemäß den Regeln der Gattung und der jeweiligen narrativen Struktur. Und auch Erzählungen erbringen solche Stabilisierungsleistungen im Modus unvollständigen Wissens -- entweder weil vollständiges Wissen grundsätzlich nicht zu erhalten ist oder weil es 
zu aufwändig wäre, es zusammenzutragen. Denn erzählılt wird ja nicht nur da, wo jemand ein Wissen mitteilt, sondern auch um Mangel an Wissen zu bewältigen oder zu ignorieren.

Erzählen ist eine universelle kulturelle Praktik der Summierung, Schematisierung, Reduktion und des Spiels mit komplexen Zusammenhängen unter Bedingungen unvollständigen Wissens. Narrative Schemata filtern unübersichtliche Datenmengen aus, stellen Selektionszwänge her und sichern sowohl kognitive Wiedererkennbarkeit als auch kommunikative Redundanz. Wie Institutionen bringen Erzählungen abgeschirmte Eigenzeiten und -räume, allerdings um den Preis der Deformation des durch sie geschaffenen Weltzugangs. Als , ExklärungsHeuristiken<, die mit begrenztem Aufwand an Informationsverarbeitung Plausibilitäten herstellen, über die sich leicht Konsens erzielen lässt, leiten sie das Verhalten von søzialen Akteuren, ja von ganzen Gesellschaften an. So zählen sie zu den von North genannten Vermittungsinstanzen, die Entscheidungsprozesse vorbestimmen und kanalisieren. Und wie im Fall von Institutionen schafft die Entscheidung für ein Narrativ - etwa die Wir Erzählung einer Ethnic oder Nation -..-Pfactabhängigkeiten, aus deneu zu eineu späteren Zeitpunkt, vor allem im Fall eines Konflikts, nur noch schwer herauszukommen ist. Ob wahr oder falsch, Narrative gewähren subjektive Erwartungssicherheit, die sich durch selektive Folgewahmehmungen beziehungsweise die Interpretation solcher Wahmehmungen in der Regel bestätigt, also den Mechanismus ihrer Selbststabilisierung gleich mitliefert. Der Wert dieser Stabilisierung scheint so hoch, dass sich Narrative ein Paradebeispiel dafür wären Konspirationstheorien - sogar durch gegenläufige Tatsachen selten erschüttern lassen.

Wegen seiner Indifferenz gegenüber der Alternative wahr/falsch ist clas Erzählen als eine Erkenntnisform minderen Ranges diskreditiert worden .... als bloße Entwicklungsstufe auf dem Weg zu richtigerem, vollständigerem, präziserem, rational kontrolliertem Wissen. Folglich müsste die Menschheit auf dem Weg zur vollkommenen Aufklärung das Erzählen wie eine archaische Kulturtechnik hinter sich lassen. Aber ein solches Programm der, Überwindung des Mythos< provoziert eine Reihe von Gegenfragen: Ist das Erzählen nur dort sinnvoll und legitim, wo man $>$ nicht alles weiß? Gibt es ein solches totales Wissen überhaupt, und wenn ja, auf welchen Feldern? Steht nicht jeder kognitive und kommunikative Akt vor der Notwendigkeit, Komplexität zu reduzieren, und muss sich folglich auf kulturelle Reduktionstechniken stützen? Ist ein >Modemozentrismus< aufrechtzuerhalten, der in älteren beziebungsweise außermodernen Kulturen ein mythologisch verbrämtes Unwissen ansiedelt, während die Errungenschaften der Moderne sich ihrer Kraft zur Entmythologisierung verdanken? Nimmt Nichtwissen in dem Maß̈ ab, in dem Wissen zunimmt? Wenn man Zweifel hat, all diese Fragen vorbehalt los zu bejahen, dann erscheinen Semiotechniken wie das Erzählen nicht mehr als bloße Vorstufe zu einem aufgeklärten, wissenschaftlich purifizierten Wissen. In Gegenteil, sie sind in dem Maß unersetzlich (und unüberwindbar), in dem unvollständiges Wissen konstitutiv für die Organisation von sozialen Zusammenhängen ist tund trotz aller Erkenntnisfortschritte im Einzelnen gesellschaftlich immer wieder regeneriert wird.

4.

Man muss noch einige Zusatzhypothesen einführen, um die vollen Konsequenzen aus dem hier vorgefuihrten Ansatz zu ziehen. Was mir dabei als Ziel vor Augen schwebt, ist eine kulursemiotiscbe Feldtheorie, die in ihrer Anlage vor allem durch die Arbeiten von Jurij Lotman inspiriert ist. Lotman verwendet für kulturelle Kommunikationstäume den Begriff der Semiosphäre, und er sieht diese Semiosphären durch mehrere für unseren Zusammenhang wichtige Eigenschaften bestimmt: Sie sind anisotrop, weisen eine ungleichmäßige Dichte auf und bergen in sich ein vielfältiges Strukturgefäle zwiscben Zentren und Peripherien - und zwar in dem Sinn, dass semiotische Gebilde in ihrem jeweiligen Zentrum rigide und in ihren Bedeutungszuweisungen festgelegt sind, sich aber an den Rändern in Mehrdeutigkeiten und Unbestimmtheiten auflösen.

Dieses Raummodell lässt sich unschwer mit einem Modell der diskontinuierlichen Verteilung semantischer Energien verknüpfen. Dabei ist dreierlei zu beachten. Erstens sind der Aufbau und die Pflege einer in sich konsistenten Semantik zeit- und energieintensiv. Zweitens ist der Raum der Semiosphäre ein widerstän. diger Raum. Auf ähnliche Weise wie politische Machträume, die häufig die Direktiven aus dem Machtzentrum in die Provinzen verpuffen und die Befehlsketten im Nirgendwo enden lassen, sind auch der Ausbreitung von Semantiken über physische und symbolische Distanzen hinweg Grenzen gesetzt -- es sei denn, ihnen kommen eine geeignete Infrastruktur und die nötigen sozialen Trägerkräfte zu Hilfe. Drittens sind Übersetzungswiderstände zwischen verschiedenen Semantiken oder auch nur zwischen unterschiedlichen Operationsebenen innerhalb derselben Semantik einzuberechnen.

Wenn die Welt der Zeichen anisotrop ist, dann haben Sinnbezinke, Rationali.. täten und logische Stimmigkeiten jeweils nur eine regionale Reichweite, und innerhalb dieser Reichweite wiederum nur einen begrenzten Schärfebereich. Aus der hier angedeuteten kultursemiotischen Perspeltive ist kein Sinnsystem, gleich welcher Art, in sich volkommen geschlossen, selbstidentisch und kohärent. In seiner Mitte mag sich die Kohärenz verdichten, aber an seiner Peripherie läuft es gewissermaßen in lose Enden aus, an denen seine Codes nicht oder nicht meh: in vollem Umfang greifen. Jedes Gedankengebilde, jedes Argument, ja sogar jeder Begriff grenzt an undeutliche, unausgeleuchtete Zonen, die bei Licht besehen einen Keim der Unstimmigkeit in das System tragen. Aber diese Unstimmigkeit 
bleibt in der Regel latent, weil es zur ökonomischen Eimrichtung und Balance solcher semiotischen Gebilde gehört, dass sie ihre unscharfen Ränder gerade nicht scharf stellen, sondern unartikuliert und vage belassen. Systematisierung kostet (Gedanken-)Zeit und Energie, weil sie stillschweigend oder unerkannt Inkonpatibles, Ungeklärtes, schwach defunierte Begriffe, Vieldeutigkeiten etc. in manifeste logische Störungen und Widersprüche verwandelt und dadurch eine Art von Regelungs- beziehungsweise , Nachrüstungs bedarf auslöst. Aber erstens gibt es kein in diesem Verständnis widerspruchsfreies und vollständiges System -- nicht ein mal in der Mathematik. Und zweitens wäre es für ein System (in diesem Fall enger zu fassen: ein epistemisches Regime) vollkommen unwirtschaftlich, pausenlos zu versuchen, seine Raudbedingungen scharf zu stellen.

Anders formuliert: Logische Konsistenz in ihrer positiven (Widerspruchsfreiheit) oder negativen Form (als herauspräparierter, artikulierter Widerspruch) ist nur im Hochenergiemodus semiotischer Systeme zu haben, Sie beruht auf Denkzwang (durch Nicht-Tolerierung von Abweichungen, Elastizitäten, Mehrdeutigkeiten, unentschiedene Zonen), der nicht von selbst in der Welt ist und auch kein Korrelat in der Wirklichkeit hat, sondern auf einer langen Kette von Voratsset zungen beruht: auf himeichender Muße; professioneller Schulung; Ausbildung von Fachsprachen, die nach einem Ausdruck von Flans Blumenberg »exklusive Regionalidiome sind; ${ }^{18}$ auf Medien, Aufzeichnungssystemen und Archiven; und nicht zuletzt auf institutioneller Gintergrundstabilität. Denn der Gedanke, dass logische Konsistenz unwabrscheinlicb ist und hohe Kosten verursacht, verknüpft die Epistemologie wiederum mit der Institutionentheorie. Insbesondere ist hier Gehlens Theorem der Entlastung durch Institutionen in Anschlag zu bringen, Institutionen regeln nicht nur die Energiezufuhr zur dauenden Wiederherstellung logischer Konsistenz (zum Beispiel in den Wissenschaften oder im Recht), sondern sie erlauben auch Einsparungen - und zwar gerade dort, wo es un die losen Enden von epistemischen Ordnungen geht. Sie schützen nämlich nicht nur die Gedanken (durch Verfestigtng und Verstetigung), sondern auch die Gedankenlosigkeiten, die so etwas wie Pufferzonen zwischen inkompatiblen Semantiken bilden. Würde die Stabilität eines Systems nur von seiner sinnhaften Kohärenz abhängen, wäre seine Semantik hoffnungslos überfordert. Wie ich an anderer Stelle genauer ausgeführt habe, ist sinn ein aufwändiges, kostenintensives Register der Systemstabilisierung. ${ }^{19}$ Hoher argumentativer Aufwand wäre nötig (und nie wirklich erfolgreich), um das System gegen Verwerfungen an seinen Rändern zu immunisieren.
Aus der klassischen Logik kennt man Begriffsbäume, die in hierarchischer Stufung vom Einzehen und Partikutaren zum Allgemeinen hinaufreichen, Nach cliesem Stufenprinzip sind nicht nu einzelne Deduktionen, sondern ganze metaphysische Wissenssysteme angeordnet. Auf das Problem sozialer Klassifikationen bezogen, würde ein solcher Baun bei den Individuen anfangen und über lokale Gemeinschaften, Ethnien, Nationen und (in bestimmten kulturellen Semantiken) Rassen in aufsteigender tinie schließlich in der Menschheit als dem umfassendsten Kollektivbegriff gipfeln.

In einem solchen Begriftsbaun wäre dem Prinzip nach jede untere Einheit je weils subsumptionslogisch in der jeweils übergeordneten Einheit enthalten und würde in ihr - im Hegelschen Doppelsinn --atfgehoben. Welche akzidentiellen Eigenschaften auch immer ein Individuum hat, es ist in bestimmten Verbänden organisiert, gehört einer ethnischen Gruppe an, die wiederum in einer Nation politisch formiert ist, es zeigt Mertmale einer bestimmten Rasse und ist auf der allgemeinsten Stufe der Prädikation ein menschliches Wesen. Kurz gesagt, die Akzidentien der logisch inferioren Begriffsebene heben sich in den substanziel an Wesensbestimmungen auf der jeweils höheren lbene auf. So wohnt zum Beispiel der Begriff des Menschen dem Begriff des besonderen Individuuns inne und kann logisch aus ihm deduziert werden; diese Deduktion ist zwingend und kostet, so scheint es jedenfalls, keine gedankliche oder hermeneutische Energie. Die logische Stufenfolge wäre demnach in einen sleeren, linear, ohne Zeitverlust und Energieaufwand zu durchquerenden Raum eingebettet. Streng genommen ist aber auch diese räumliche Metapher unbrauchbar, denn der Unterbegriff is ja im Oberbegriff schon enthalten, so dass zwischen beiden keine Entfernung durchatert werden muss. Rein logische Prozesse spielen sich mithin ohne Me. dium, jedenfalls ohne widerständiges Medium ab.

Wenn man die gleiche Stufenfolge nun aber aus kultursemiotischer Perspektive betrachtet, dann gelten ganz andere Voraussetzungen. Aus dieser Perspek. tive ist jeder Schritt von einer Ebene zur anderen eine Übersetzung, die ihren jeweiligen Inhalt transformiert. Keine dieser Subsumptionen geht glatt auf; in der Richtung von unten nach oben nicht, weil nicht alle Merkmale auf die nächsthöhere Stufe gehoben werden; in der Richtung von oben nach unten nicht, weil die jeweiligen oberbegriffe ihrer je eigenen Logik gehorchen und den Charakter der Elemente, aus denen sie bestehen, verändern beztehungsweise ihren Sinn verschieben. In den Begriff der lokalen Gemeinschaft gehen nicht alle Figenschaf ten der Individuen ein; es bleibt also ein Rest, der sich der Subsumption nicht unterwirft. Umgekehrt werden andere Eigenschaften erst durch die Zugehörigkeit zur lokalen Gemeinschaft geschaffen oder hervorgetrieben: die Subsumption is 
also nicht nur unvollständig, sondern greift ihrerseits in den Bestand der zu sub. stmierenden Elemente ein. Und das wiederholt sich von Stufe zu Stufe.

Dazu kommt, dass in kultursemiotischer Perspektive der Weg die Stufenleitet hinauf und hinunter nicht ohne einen doppelten Aufwand zurückgelegt werden kann: einen Aufwand an Zeit und an Energie. Dent mit jeder Niveaustufe ändert sich die gesamte Grammatik, nach der die jeweiligen Kategorien funktionieren. Es kostet eine gewisse Mühe, die jeweilige Grammatik sozial zu implementiexen und sozusagen zu betreiben<. Sie erfordert Abstraktions- und Systematisierungs leistungen und bedarf deshalb der Kohärenzpflege durch dafür geeignete Institutionen - denn solche Leistungen sind nicht setbstevident, sondern auf Exper ten, Deutungsexperten, angewiesen, die sich mit der nötigen infrastrukturellen Unterstützung um die systempfleger kümmern.

Ein Gedankenexperiment kann zur Veranschaulichung dienen: Man stelle sich isoliert voneinander lebende lokale Gemeinschaften vor, zum Beispiel unte den Indianern Nordamerikas in frühen 19. Jahthundert. Diese lokalen Gemeinschaften werden über ein bestimmtes ethnisches Bewusstsein verfigen; sie werden sich einem Stamm zugehörig fühlen. Von Nationen euxopäischer Prägung haben sie nicht viel gehört, außer von den Vereinigten Staaten. Dass die gesamte Menschheit in Nationen mit ihren jeweiligen Territorien aufgeteil ist, käme th nen überhaupt nicht in den Sinn. Auch ein allgemeines Bewusstsein von Rassen zugehörigkeit werden sie nicht entwickelt haben, abgesehen von dem offenbaren Konflikt zwischen Weißen und sogenannten Roten. Erst recht werden sie über kein abstraktes Konzept von Menschheit verfügen - zumal in vielen Stammessprachen der Ausdruck für >Mensch oder $>$ Menschenwesen< für die eigene we group reserviert ist. Nun können natürlich Missionare zu ihnen gelangen und ihnen erklären, dass sie Angehörige einer ihren Gesichtskreis und Aktionshorizont weit überschreitenden Menschheit sind, dass Gott mit dieser Menschheit insgesamt in Verkehr steht (und nicht bloß eine Stammesgotheit ist) und dass vor Gott alle Menschen gleich sind. Aber das werden für sie eben sehr abstrakte sozial weitgehend inoperable Begriffe sein; Begriffe, die von ihrer Handhabung durch geschulte Deutungsexperten abhängig sind und sich ohne derer Mithilfe schnell ins Nebulöse ve:lieren. Um Allgemeines nicht nur zu denken, sondern auch sozial wirksam zu machen, bedarf es eben solcher geschulter Dettungs experten; der Institutionen, die sie hervorbringt; der Infrastruktur, die sie ver kehrsfähig hält; einer Macht, die ihren Schirm über sie ausbreitet. Ohne diesen enormen sozialen Aufwand wird eine Reflexion, die solche Subsumptionsketten durchläuft, nicht auf Dauer zu stellen sein.
6.

Eine Beschreibungsweise für die Funktionsweise von Zeichensystemen, die ökonomische beziehungsweise energetische Faktoren einbezieht, ist eingestandenermaßen ohne einen erheblichen zusätzlichen Aufwand nicht zu erreichen. Die leitende Prämisse ist, dass der Raum, der kulturell durchmessen wird, nicht leer, sondem dick, nicht linear, sondem vielfach gefaltet oder gerastert ist und dass alle Operationen in diesem Raum Zeit und soziale Anstrengung fordern. Der Ubergang von einer Ebene zur anderen ist nicht mit der Mühelosigkeit des Gedankenflugs zu vollziehen, sondern ist mit der ganzen Schwere eines Wechsels von einer Machtgrammatik in eine andere betastet. Denn nicht nur zwischen unterschiedlichen Semantiken, auch innerhalb desselben Zeichensystems gilt das Gesetz einer zumindest partiellen Unübersetzbarkeit zwischen den unterschiedlichen Begriffsebenen.

Die Dinge werden noch komplizierter, wenn man sich vor Augen hält, dass abstrakte oder universelle Kategorien, sobald sie sozial wirksam werden, einen ihrer Operationshohe entsprechenden Systemzwang produzieren. Das äußert sich zum Beispiel darin, dass sie Widersprüche erscheinen lassen, die auf der Ebene segmentärer, jeweils lokal begrenzter Begriffichkeiten nicht wahrnehmbar sind oder inaktiv bleiben. Es mag wie eine rein logische Deduktion aussehen, die den Begriffen nichts hinzufügt und nichts wegnimmt, sondern bloß entbirgt, was sie enthalten, wenn man folgendermaßen argumentiert: In jedem Individuum, so partikular es sein mag, steckt das Gattungswesen Mensch, dessen Eigenschaften von der Natur gegeben sind und sich durch alle individuelle Besonderheiten hindurch auffinden lassen. Aber wenn diese Einsicht sozial operativ wird, etwa in dem Satz: »Alle Menschen sind von der Natur gleich geschaffen «, oder: "Alle Menschen sind gleich geboren«, dann treibt ein so schlichter logischer Schluss Widersprüche zwischen sozialen Regelungen hervor, die bis dahin sozusagen unaufgerechnet nebeneinander bestanden hatten. Nicht immer werden solche Widersprüche unmittelbar virulent; sie genießen oft noch über lange Zeit eine Art von Latenzschutz, obne doch wirklich zur Ruhe zu kommen. Im Zeichen des Gleichheitsideals konnte sich in Amerika eine Demokratie etablieren, die zum Teil aus Sklavenhaltem bestand; und in der Französischen Revolution wurde die Forderung erfolgreich abgewehrt, die Idee der égalité auch auf das Verhältnis der Geschlechter auszudehnen. Im einen Fall hat das Unterkonzept $>$ Rasser erfolgreich der vollen und konsequenten Durchsetzung des Konzepts >Menschheit widerstanden; im anderen Fall hat sich ein Relikt segmentärer Gesellschaftsordnungen - die Idee des Haushalts, dessen Mitglieder dem männlichen Haushaltsvorstand als dem einzigen vollgültigen Rechtssubjekt untersteben - in den Universalismus der republikanischen Neuordnung Europas eingeschrieben. 
An diesen Beispielen erkennt man zum einen, wetcher Widerstand logischen Konklusionen im kulturellen Feld entgegenwirken kaur, In cinem kultursemiotischen Szenario, wic es hier umrissen wurde, funktionieren Begriffe nicht einfach nach den Regeln der Induktion und der Deduktion; sie sind, schon auf der einfachsten Stufe, heterogene und polymorphe Komplexe, die in Relation zu Prakti. ken, Vergangenheiten, spezifischen sozialeu Dynamiken, Sinnladungen aller At stehen. Jeder Ebenentransfer, jede Transposition in einen anderen sozialen Maß stab verändert sie und unterstellt sie neuea Koordinaten; auf jeder Bbene geiten eigene Aktivierungsmuster, die bestimmte Sinnkomponenten privilegieren und andere in die Latenz zwingen. - Ganz abgesehen davon, dass man es nie mi einem einzigen Begriffsbaum zu tun hat, sondern mit vielen Bäumen, die teil weise ineinander verwachsen, teilweise ganz voneinander getrennt sind und viel leicht nicht einmal in einer sozial wahrnehmbaren Beziehung zueinander stehen.

Zum zweiten sollte aber auch dentlich geworden sein, worin die kulurelle Funktionaliät von logischer und informationeller Unvollständigkeit liegen könnte. Nicht nur ist Konsisten\% über große Felder hinweg unwahrscheinlich weil zeit- und energieraubend; sie wäre von einem bestimmten Punkt an sogar dysfunktional, weil sie Sinusysteme start werden ließe und Gesellschaften auße Stand setzen würde, zwischen ungleichnamigen Prinzipien jonglieren zu können, die bei strenger Konsistenzprüfung in hellen licht des Tages in einen offenen Wi derspruch zueinander gerieten. An solchen ,Nahtstellen des kulturellen Zeichengewebes, die immer potentielle Konfliktzonen sind, empfiehlt es sich, die Verhält. nisse im Unbestimmten zu lassen oder in die weitläufige Kunst des Nichtwissen beziehungsweise der geregelten Nichtkenntnisiahme auszuweichen. In solchen Fällen stellt der begrenzte Wissenshorizont der Akteure keineswegs nur ein Defizit dar, das sich ats dem notwendigen Energieaufwand furr die Informationsbeschaffung erklärt. Vielmehr ist darin, ob man will oder nicht, ein Funktionsprinzip von Gesellschaften zu sehen, die mit der Vielzahl ihrer potentiellen inneren Widersprüche nur leben können, indem sie es damit nicht so genau nehmenc Das kaun in Extrem zu einer kollektiven Wirklichkeitsverweigerung führen. Aber wer dagegen angehen tud die Menschen aufklärerisch wachrütteln will, sollte sich der Tatsache bewusst sein, dass er es mit Verhaltensweisen zu tun hat, die durch eine Wissensökonomie unter Knappheitsbedingungen gesteuert werden.
ANMERKUNGEN

So vor allem die in Zusammentrang der Projektgruppe PPoetologie der Körperchafenk an Berliner Zentrum für Liteaturforschung entstandeten Bücher Des Kaisers neute Kleider. Über das Imaginäre poltischer Herschaft. Texte, Bilder, Lektiren, Frankfurt an Main 2002, und Der fiktive Statt. Konstruktionen des politi schen Karpers in der Geschidine Europas, Fenkfurt an Main 2007

2 Erschienen bei Konstanz Universily Press, 2011.

3 Die folgenden Ausfihruagen über. schneiden sich mit Abschniten aus mei. ner Monographie Wabrbeit und liffindung. Grundzüge einer Aligeneinen Erzaibltheorie, Trankfurt am Main 2013, wo sic in einem noch weiträtmigeren $\mathrm{Zu}$ carmunculang entwickelt werden.

4. Joseph E. Stiglitz: : Information and the Change in the Palladigm in Ecomonics in: Econonics for an thiperfect World. lis saijs in Honor of lostpis E. Stiglits, hg. ven Richard Amott tha, Cambridge, MA Loudon 2003, S. 569- -639, dort S. 569.

5 Jakob Tanner: Hisiorische Anthropologie ztir Finfuibrung, Hambury 2004, S. 15 Dort S. 151 ff. ein knapper, aber selr hilt eicher Oberblick über die Theorieen wicklung auf diesem Feld.

6 Douglass C. North: Instiutionen, institufonelier Wanded and Wirischafisteisitum Tubingen 1992.

7 Ebd., S. 49 .

8 Fbd, S. 44 .

9 Fbd.
10 wDer Hauptzweck der lustitutionen in ciner Cesellschaft besteht darin, durch dic Schaffung einer stabilen (aber nicht uotvendigerweise effizienten) Orénung die Unsicherheit menschlicher Interak tion zu vermindern. Doch die Stabilita yon fnstitutionen steht beineswegs in Widerspruch mu der llatsabe, dab se sich verŭudern. Aus Ôbereinkünften, Ge ptogenheiten und Vechalterssormen bis hin zum gesetzten Rechi $[. . . \mid$ und Ver trägen zwischen Finzelpersonen entstehen Institutionen and verändern damit unablässig unsere Wahhuzöglichkeiten., (E.bd, S, 6)

1) Libd, S. 69 .

12. E.bed., S. 132.

13 Die Darsteliung folgt hier der Zusammenfassung bei Gerhard Roth: Persön licbkeit, Entscbetdang und Verbalten. WGa tum es so sclowierig ist, sich und andere zu ändern, Stuttgart 2007, S. 113 ff.

1.4 ibxd, S. 116.

15 Bbci., S. 118.

16 Vgl. Tanner: Historistbe Anthropologie (Anm. S), S. 153 t.

17 Ebd., S. 154.

18 Hans Blunenberg: «Sprechsittation und immanente Poctike, in: ders.: Xstbetische und metaphorologische Scbrifter, Frankfurt ant Main 2001, S. 120-135, dort S. 125.

19 Albrecht Koschorke: sNicht-Simn und die Konstitution des Serialena, in: Erlebert, Erleiden, Eiffabren. Die Konstiution soziaten Sinns jenseits instrumentelier Vernunfi, hg von Kay Jange u. a., Bielefeld 2008, S. 319-332. 